\title{
HÁBITAT LUMÍNICO, ESTRUCTURA, DIVERSIDAD Y DINÁMICA DE LOS BOSQUES SECOS TROPICALES DEL ALTO MAGDALENA
}

\author{
Light halbitat, structure, diversity and dynamic of the tropical dry forest
}

\author{
Omar Melo', Fernando Fernandez-Méndez² \& Boris Villanueva ${ }^{3}$
}

Melo, O., Fernandez-Méndez, F. \& Villanueva, B. (2017). Hábitat lumínico, estructura, diversidad y dinámica de los bosques secos tropicales del Alto Magdalena. Colombia Forestal, 20(1),19-30.

Recepción: 24 de noviembre de 2015

\section{Resumen}

Los bosques secos tropicales son ecosistemas complejos y frágiles con alta intervención antrópica y ciclos reproductivos restringidos. Albergan riqueza, diversidad estructural, fisiológica y fenológica únicas. Esta investigación se desarrolló en el valle del alto Magdalena, en cuatro fragmentos de bosque con diferentes estados sucesionales. En cada fragmento se establecieron cuatro parcelas permanentes de 0.25 ha. y se evaluó el hábitat lumínico asociado a la riqueza, abundancia relativa y rareza de especies, lo mismo que la dinámica del bosque que contempló mortalidad, reclutamiento y crecimiento diamétrico para un periodo de 5.25 años. En bosque ribereño maduro se encontró una riqueza de especies mayor a la reportada en otros estudios para áreas similares en el Valle del Cauca y la costa atlántica. Se evidencian valores de riqueza, heterogeneidad y rareza de especies mayores que los encontrados para zonas más secas del Tolima. La estructura, diversidad y dinámica del bosque fueron correlacionadas con el hábitat lumínico, mostrando diferencias en la arquitectura del dosel y su papel en la captura y absorción de radiación, Bosques con dosel denso presentan poca disponibilidad de radiación fotosintética activa en el
Aceptación: 24 de octubre de 2016

sotobosque, relacionado con baja riqueza de especies, mientras que sotobosques iluminados son más ricos y heterogéneos.

Palabras clave: coeficiente de extinción lumínico, diversidad florística, estructura, índice de área foliar, mortalidad, radiación fotosintéticamente activa, reclutamiento.

\begin{abstract}
Tropical dry forests are complex and fragile ecosystems with high anthropic intervention and restricted reproductive cycles. These have unique richness, structural diversity, physiological and phenological . This research was executed in the Upper Magdalena Valley, in four forest fragments with different successional stages. In each fragment four permanent plots of 0.25 ha were established and lighting habitat associated with richness, relative abundance and rarity of species. The forest dynamics included the mortality, recruitment and diameter growth for a period of 5.25 years. The species rischness found in the mature riparian forestis higher than that reported in other studies of similar areas in Valle del Cauca and the Atlantic coast. The values of richness, diversity and rarity species are more evidenced than
\end{abstract}

Universidad del Tolima. Ibagué, Colombia. omelo@ut.edu.co. Autor para correspondencia.

Universidad del Tolima. Ibagué, Colombia. Laboratorio de Ecológia e Evolucão de Plantas, Universidad Federal de Viçosa, MG. Brasil.fmendez@ut.edu.co.

Universidad del Tolima. Ibagué, Colombia. bsvillanuevat@ut.edu.co. 
the magnitudes found in drier areas of Tolima. The structure, diversity and dynamics of forests were correlated with the lighting habitat, showing differences in canopy architecture and its role in the capture and absorption of radiation. Forests with dense canopy have limited availability of photosynthetically active radiation in understory related low species richness, while illuminated undergrowth are richer and heterogeneous.

Key words: lighting extinction coefficient, floristic diversity, structure, leaf area index, mortality, photosynthetically active radiation, recruitment.

\section{INTRODUCCIÓN}

El bosque seco tropical se caracteriza por presentar al menos una estación de sequía al año, la cual genera déficit hídrico en el suelo con las consecuentes alteraciones en la funcionalidad de la vegetación. De tal manera, se genera defoliación en gran cantidad de especies que se han adaptado a la sequía bajo esta estrategia. Sin embargo, otro grupo de especies permanecen siempre verdes, para las cuales la estrategia adaptativa obedece a la eficiencia y control de la conductancia estomática (Murphy \& Lugo, 1996; Lobo et al., 2003; Luttge, 2008). La diversidad que constituyen los bosques secos tropicales es más simple que la presentada en los bosques lluviosos tropicales y en los bosques andinos. Sin embargo, su valor radica en el alto número de endemismos que pueden alcanzar entre $43 \%$ y $73 \%$ que, junto con sus bajas tasas de crecimiento, lo clasifican como ecosistema de alta fragilidad. Igualmente, es típica de este ecosistema la complejidad fisiológica de las especies y los patrones de distribución espacial de las especies y sus poblaciones (Janzen, 1986; Mooney et al., 1996; Burnham, 1997; IAVH, 2002). La estructura y el patrón de la diversidad de los bosques secos tropicales son contrarios al de los bosques lluviosos, los cuales incrementan la riqueza de especies con su proximidad al ecuador. La mayor densidad de especies en el bosque seco tropical se ubica en áreas más septentrionales que ecuatoriales (Chazdon \& Denslow, 2002; Gentry, 1995; Kalacska et al., 2004).

El factor limitante de los bosques secos tropicales es la disponibilidad del agua en el suelo, por lo cual los procesos de lixiviación de nutrientes se encuentran restringidos. Lo anterior ha generado a nivel mundial el cambio del uso del suelo en estos ecosistemas hacia la agricultura y la producción ganadera. Para esto se han formado estrategias de producción agrícola y pecuaria basadas en sistemas de riego que incrementan la producción de alimentos, afectando drásticamente el flujo de los servicios ecosistémicos al destruir las coberturas naturales y ser remplazadas por paisajes antropizados (Bazzaz \& Pickett, 1980; Quesada \& Stoner, 2004; Montagnini \& Jordan, 2005).

Los bosques secos tropicales colombianos se ubican especialmente en dos grandes regiones que corresponden a la llanura caribe y los valles interandinos de los ríos Cauca y Magdalena. En estos la precipitación es inferior a los 2000 mm anuales. Sin embargo, la distribución de la precipitación determina las características particulares en la vegetación. La Ilanura Caribe tiene clima diagramas monomodales mientras que los valles interandinos de los ríos Cauca y Magdalena son bimodales, adicionados al efecto sombra de las cordilleras (Repizzo \& Devia, 2008; Linares \& Fandiño, 2009). En cuanto a las coberturas naturales actuales en el Alto Magdalena estas se ubican tanto en el norte y sur del departamento del Tolima, como en el norte y sur del departamento del Huila. La subregión con menor frecuencia de fragmentos naturales de bosque seco tropical y de menor tamaño corresponde al sur del Tolima-norte del Huila, en la cual la cobertura natural no supera el $2 \%$. Fragmentos con tamaños superiores a las 200 ha y con mayor grado de conservación se encuentran en el norte del departamento del Tolima y con coberturas 
superiores al 7 \% del área original (Etter, 1993; IAVH, 2002; Universidad del Tolima, 2002). Los resultados de investigación en cuanto a estructura y diversidad de los bosques secos tropicales son escasos, y es más crítico aún para los valores de su dinámica. Tan solo en la actualidad comienzan a conocerse los primeros resultados del conocimiento de la funcionalidad de este tipo de bosque, lo que lo ubica como uno de los ecosistemas más degradados, con un nivel de alta vulnerabilidad y con vacíos de conocimientos que permitan una verdadera conservación, restauración y uso sostenible de sus servicios ecosistémicos (Sanchez-Azofeifa et al., 2005; Prance 2006).

Por lo anterior, el presente estudio se realizó en las áreas de bosque seco tropical ubicadas en el norte del departamento del Tolima, que hacen parte de la ecorregión del Alto Magdalena. Con el objeto de determinar la relación que existe entre el hábitat lumínico que genera el dosel del bosque (oferta de radiación fotosintéticamente activa en el entorno de crecimiento) y la estructura, diversidad florística y la dinámica de las coberturas en cuanto a crecimiento, mortalidad y reclutamiento, en cuatro bosques secundarios con diferente estado sucesional que se ubican en el flanco oriental del piedemonte de la Cordillera Central y hacen parte del complejo de bosques secos tropicales del valle geográfico del río Magdalena.

\section{MATERIALES Y MÉTODOS}

\section{Área de estudio}

Esta investigación se desarrolló en cuatro fragmentos naturales de bosque seco tropical (bs-T), con diferentes estados sucesionales, localizados en la parte alta del valle geográfico del río Magdalena, en terrenos pertenecientes al Centro Universitario Regional del Norte (Curn) de la Universidad del Tolima, en el municipio de Armero-Guayabal, al norte del departamento del Tolima. Los bosques seleccionados hacen parte de la vertiente del río Magdalena y se ubican en el piedemonte del flanco oriental de la Cordillera Central y la Ilanura aluvial. Las coordenadas planas de la zona son: $4^{\circ} 59^{\prime} 53.48^{\prime \prime} \mathrm{N}$ y $74^{\circ} 55^{\prime} 38.87^{\prime \prime}$.

En cuanto a las características climáticas, el área de estudio tiene una temperatura media anual de $27^{\circ} \mathrm{C}$, una precipitación promedio de $1750 \mathrm{~mm}$ y una humedad relativa del $71 \%$. La altura sobre el nivel del mar oscila entre los 475 a 580 m de altitud. La evaluación climática muestra un comportamiento bimodal, con un primer período de lluvias entre los meses de marzo a mayo y un segundo período más fuerte entre septiembre y noviembre (Fernández et al., 2014).

\section{Muestreos}

Las coberturas objeto de evaluación corresponden a cuatro bosques con diferente estado sucesional. El primero es un bosque secundario temprano (BST) de 10 años de edad, originado a partir de fuegos antrópicos y dominado por la asociación Curatella-Xilopia. El segundo tipo de bosque corresponde a un bosque secundario en recuperación (BSR) de 20 años de edad, generado por abandono de la actividad de ganadería extensiva y dominado por Cordia alliodora (Ruiz \& Pav.) Oken. El tercero corresponde a una sucesión en estado de recuperación avanzada de más de 40 años y que para efectos del presente estudio se denominará bosque secundario maduro (BSM), con una composición florística heterogénea. El cuarto tipo de bosque corresponde a un bosque ribereño maduro (BRM), dominado por Anacardium y Ceiba cuyo tiempo de recuperación supera los 60 años. En cada uno de los bosques se establecieron cuatro unidades de monitoreo permanente de 0.25 has (50x50 m), con subparcelas de 10x10 m, para un área total de muestreo por tipo de cobertura de 1 ha. Este seccionamiento de la unidad muestral obedeció a las limitaciones por tamaño y forma de los fragmentos remanentes. 
Para todas las unidades de muestreo se registraron, marcaron, midieron y colectaron todos los individuos con diámetro normal mayor e igual a 5 cm. Las mediciones del diámetro normal se realizaron con cinta diamétrica con aproximación al milímetro (Melo \& Vargas, 2003). La colección del material vegetal se herborizó y determinó con la colaboración del herbario del Jardín Botánico Joaquín Antonio Uribe de Medellín y el laboratorio de dendrología de la Universidad del Tolima. El primer inventario se realizó en junio 2009, con posteriores monitoreos anuales hasta septiembre de 2014, para un intervalo de tiempo de 63 meses (5.25 años). Los nuevos individuos que superaron los $5 \mathrm{~cm}$ de diámetro normal se registraron como ingresos o reclutados y a los árboles muertos se les registró la condición de mortalidad (Phillips, et al., 2005).

\section{Estructura y diversidad}

Para la evaluación de la diversidad florística y de los parámetros estructurales convencionales se utilizó el programa Stimate-S 9.1.0 (Colwell et al., 2012), a nivel intracomunitario se seleccionaron medidas de abundancia de especies (riqueza, heterogeneidad relativa y rareza de especies) y a nivel intercomunitario se utilizaron medidas de similaridad y disimilaridad (Magurran, 1998; Magurran 2004; Magurran \& McHill, 2011).

\section{Dinámica del bosque}

La evaluación de la dinámica para los cuatro tipos de bosque contempló el cálculo de la mortalidad, el reclutamiento y el crecimiento diamétrico para el periodo de evaluación de 5.25 años. La tasa de mortalidad se determinó a partir del modelo propuesto por Castro et al. (2005) y el reclutamiento según los modelos expuestos en Melo \& Vargas (2003). El patrón de mortalidad contempló los tipos de muerte: tronco partido (TP), caído de raíz (CR), individuos desaparecidos (DE) y cortado
(COR) (Carson \& Schnitzer, 2008). El crecimiento a partir de la aplicación de modelos determinísticos convencionales (Weiskittel, 2011).

\section{Habitat lumínico}

Para evaluar el hábitat lumínico que indica la cantidad de energía utilizada por la cobertura boscosa para realizar los procesos funcionales de asimilación y productividad se empleó la medida directa del índice de área foliar (IAF), con un analizador de fronda vegetal LI-2200TC. Los diferentes niveles de luz absorbidos por el dosel de los bosques objeto de evaluación fueron expresados en valores relativos de la radiación fotosintéticamente activa (RFA), la cual fue medida como la radiación no absorbida sobre la superficie del suelo del bosque expresada como la densidad de flujo de fotones fotosintéticos $\left(\mu \mathrm{mol} . \mathrm{m}^{-2} . \mathrm{s}^{-1}\right)$, entre los 400 y $700 \mathrm{~nm}$ del espectro lumínico, para lo cual se utilizaron los sensores LI-191SA-Line Quantum Sensor y LI190SA-Quantum Sensor y un colector de datos LI 1400 (LI-COR Inc., Lincoln, NE. USA). Igualmente, se derivó el coeficiente de extinción de la luz (K) mediante la aplicación de la ley de Beer \& Lambert (Lüttge, 2008; Pallardy, 2008). Este proceso se realizó por debajo del dosel sobre la superficie del suelo en el centro de cada subparcela de 10x10 $\mathrm{m}$, en el cual se registraron cuatro lecturas cardinales. Las mediciones se realizaron entre las $10 \mathrm{y}$ las 14 horas.

\section{RESULTADOS}

En la tabla 1 se muestran los resultados para las variables respuesta por niveles de evaluación (estructura, diversidad florística, dinámica y hábitat lumínico), para los cuatro tipos de bosque secundario en el alto Magdalena (BST: bosque secundario temprano, BSR: bosque secundario en recuperación, BSM: bosque secundario maduro, BRM: bosque ribereño maduro). 
Tabla 1. Comportamiento de la estructura, diversidad, dinámica y hábitat lumínico en cuatro tipos de bosque seco tropical en el alto Magdalena.

\begin{tabular}{|c|c|c|c|c|c|}
\hline \multirow{2}{*}{$\begin{array}{c}\text { NIVEL DE } \\
\text { EVALUACIÓN }\end{array}$} & \multirow{2}{*}{ VARIABLES } & \multicolumn{4}{|c|}{ BOSQUES } \\
\hline & & BST & BSR & BSM & BRM \\
\hline \multirow[t]{9}{*}{ Estructura } & NA & 1774 & 928 & 388 & 672 \\
\hline & S & 18 & 27 & 36 & 48 \\
\hline & G & 21.9 & 18.7 & 17.7 & 34.2 \\
\hline & dmax. & 27.5 & 55 & 65 & 110 \\
\hline & DMg & 2.27 & 3.81 & 5.87 & 7.22 \\
\hline & $\mathrm{DMn}$ & 0.43 & 0.89 & 1.83 & 1.85 \\
\hline & $\mathrm{H}^{\prime}$ & 1.38 & 2.34 & 2.76 & 2.98 \\
\hline & E & 0.61 & 0.69 & 0.83 & 0.81 \\
\hline & $1 / \mathrm{D}$ & 4.78 & 5.30 & 11.24 & 10.98 \\
\hline \multirow{8}{*}{ Diversidad } & $1 / d$ & 2.73 & 2.97 & 5.11 & 8.84 \\
\hline & $\mathrm{CH} 1$ & 22.25 & 21.37 & 36.28 & 42.79 \\
\hline & Ab1 & 3 & 5 & 11 & 14 \\
\hline & Ab2 & 2 & 4 & 7 & 6 \\
\hline & $U$ & 2 & 3 & 5 & 5 \\
\hline & Uab. & 0 & 1 & 4 & 4 \\
\hline & Alfa & 4.45 & 9.21 & 17.67 & 18.21 \\
\hline & TMC & 2.37 & 1.37 & 0.63 & 0.45 \\
\hline \multirow[t]{3}{*}{ Dinámica } & $\mathrm{M} \%$ & 5.78 & 3.45 & 2.31 & 1.87 \\
\hline & $\mathrm{R} \%$ & 3.8 & 3.2 & 2.1 & 1.2 \\
\hline & $\mathrm{IAF}$ & 8.3 & 5.1 & 4.5 & 3.1 \\
\hline \multirow[t]{2}{*}{ Hábitat lumínico } & K & 0.75 & 0.63 & 0.53 & 0.49 \\
\hline & RFA & 16.3 & 18.2 & 22.2 & 25.7 \\
\hline
\end{tabular}

BST: bosque secundario temprano. BSR: bosque secundario en recuperación. BSM: bosque secundario maduro. BRM: bosque ribereño maduro. NA: Número de árboles. G: Área basal en $\mathrm{m}^{2}$.ha ${ }^{-1}$. Dmáx: diámetro máximo del fuste en $\mathrm{cm}$. S: número de especies. DMg: riqueza de especies de Margalef. DMn: densidad de especies de Menhinick. H’: Diversidad de Shannon. E: uniformidad de Shannon. 1/D: Heterogeneidad de Simpson. 1/d: dominancia de Berger Parker. CH1: rareza de especies. Ab1: Especies con un solo individuo. Ab2: especies con dos individuos. U: especies representadas en una sola parcela. UAb: especies únicas en una parcela. Alfa: diversidad de especies. IAF: índice de área foliar. K: Coeficiente de extinción lumínica. RFA: radiación fotosintéticamente activa. TMC: tasa media de crecimiento. M\%: tasa anual de mortalidad. R\%: tasa anual de reclutamiento.

\section{Estructura}

En cuanto a la estructura de los bosques se muestra el número de árboles (NA) en valores absolutos y el área basal $(\mathrm{G})$ en $\mathrm{m}^{2}$.ha-1 y el diámetro máximo del fuste (dmáx) en cm. En general, la estructura diamétrica de los bosques mostró tendencias de J invertida o L, típica de las coberturas dinámicas. El estado de desarrollo de cada bosque se manifiesta en la disminución del rango diamétrico, de tal manera que en las coberturas con sucesiones tempranas el rango es corto $(27.5 \mathrm{~cm})$ en comparación con las coberturas de edades más avanzadas que superan los $60 \mathrm{~cm}$. Para el tipo BRM se aprecia una tendencia bimodal que corresponde a la presencia de dos poblaciones de árboles, la primera con rangos diamétricos hasta de $35 \mathrm{~cm}$ y una segunda población cuyo rango diamétrico varía entre 45 y $110 \mathrm{~cm}$. La acumulación del área basal alcanza el mayor valor en BRM. Sin embargo, es claro el efecto que tiene el tamaño poblacional (1774) en G (21.99) para el BST. La tendencia de la acumulación del área basal (G) para los bosques secundarios es típica de una crono-secuencia, de tal forma 
que a medida que el bosque avanza en la sucesión el tamaño poblacional disminuye y los individuos alcanzan un mayor tamaño (figura 1a). El BRM manifiesta una distribución más suavizada que indica uniformidad de las poblaciones en el rango de tamaño. En la acumulación del área basal (figura 1b) las tendencias son diferenciales y contrarias a medida que avanza la sucesión, de tal manera que en el BST la mayor acumulación se da entre las categorías diamétricas 12.5 y $17.5 \mathrm{~cm}$, mientras que en sucesiones avanzadas (BSM) la mayor acumulación del área basal está representada en las categorías diamétricas mayores a $65 \mathrm{~cm}$ manifestando patrones opuestos.

\section{Diversidad}

En cuanto a la diversidad florística, se determinó para los cuatro tipos de bosque los valores para los índices de riqueza de especies de Margalef (DMg), densidad de especies de Menhinick (DMn), Diversidad de Shannon $\left(\mathrm{H}^{\prime}\right)$, uniformidad de Shannon (E), heterogeneidad recíproca de Simpson (1/D), dominancia recíproca de Berger Parker (1/d), rareza de especies $\left(\mathrm{CH}_{1}\right)$, especies con un solo individuo $\left(\mathrm{Ab}_{1}\right)$, especies con dos individuos $\left(\mathrm{Ab}_{2}\right)$, especies representadas en una sola parcela $(U)$, especies solo en una parcela y un solo individuo (UAb) y el parámetro de la serie logarítmica que indica diversidad de especies (Alfa). Los BRM y BSM presentan la mayor riqueza de especies ( $\mathrm{S}$, DMg) y densidades similares (DMn). Es evidente el incremento de la diversidad ( $\left.\mathrm{H}^{\prime}, \mathrm{E}, 1 / \mathrm{D}, 1 / \mathrm{d}\right)$ con el desarrollo sucesional. En cuanto a rareza de especies $\left(\mathrm{CH}_{1}\right)$, los BSM y BRM presentan los mayores valores. Sin embargo, a pesar de la homogeneidad (E) de las coberturas sucesionales tempranas (BST y BSR) estas contienen especies raras $\left(A b_{1}\right.$ y $\left.A b_{2}\right)$, lo cual incrementa su importancia para la diversidad. De acuerdo con los resultados del índice alfa de la serie logarítmica, el cual es considerado como la medida de diversidad de mayor representación biológica, el BSM y el BRM tienen valores equivalentes (17.67 y 18.21), a pesar de su diferencia estructural. La complementariedad intercomunitaria (tabla 2), para los bosques evaluados que es una
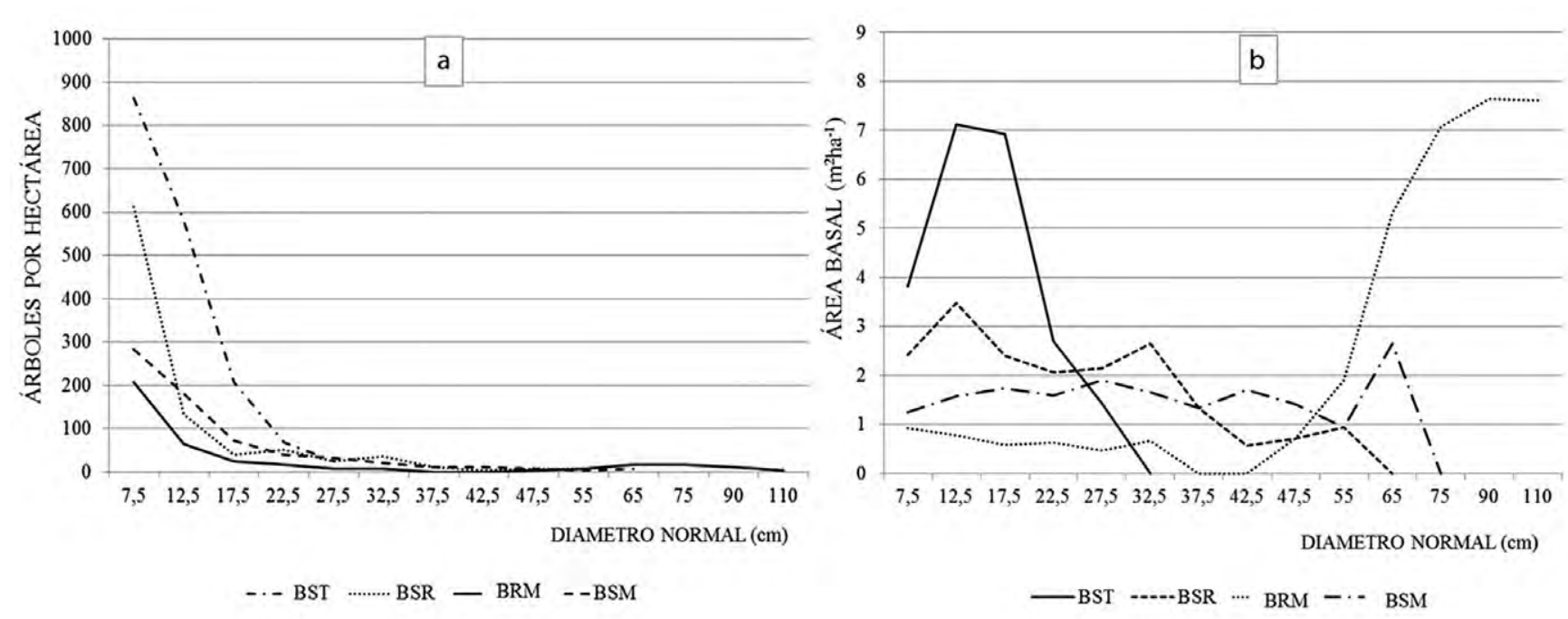

Figura 1. Tendencias de la distribución poblacional (NA) y el área basal $\mathrm{m}^{2}$.ha-1 $(\mathrm{G})$, por categorías de tamaño, para cuatro tipos de bosque secundario en el alto Magdalena. BST: bosque secundario temprano. BSR: bosque secundario en recuperación. BRM: bosque ribereño maduro. BSM: bosque secundario maduro. 
medida de betadiversidad expresada a partir de los índices de similaridad de Sorensen (SR), similaridad de Jaccard (JC), porcentaje de disimilitud (PD), porcentaje de remotidad (PR) y la distancia euclidiana (DE). Se aprecia alto valor de similaridad (SR, JC) entre BST y BSR, BSR y BSM, lo cual muestra un gradiente de acumulación de especies con el avance sucesional. Existes grandes diferencias entre la diversidad (PD, PR y DE) del BRM y los demás bosques secundarios tanto en especies compartidas como la distribución de sus abundancias.

\section{Dinámica del bosque}

En el nivel de evaluación que corresponde a los procesos dinámicos del bosque (tabla 1), se determinaron los valores de la tasa media de crecimiento (TMC), tasa anual de mortalidad ( $\mathrm{M} \%$ ) y la tasa anual de reclutamiento ( $\mathrm{R} \%$ ). Los resultados muestran una relación inversa de TCM con el avance sucesional, de tal manera que los máximos valores $\left(2.37 \mathrm{~cm}_{\text {.año }}{ }^{-1}\right)$ se logran en BST. Igualmente, se aprecia la misma tendencia tanto para $\mathrm{M} \%$ como para R\%. Las mayores tasas de mortalidad y reclutamiento (5.78 y 3.8) se manifiestan en BST como resultado de la alta competencia por recursos, contrastando con BRM (1.87 y 1.2) quien manifiesta un hábitat más estable.

\section{Hábitat lumínico}

El alto valor del índice de área foliar (IAF, $8.3 \mathrm{~m}^{2}$ área follaje $/ \mathrm{m}^{2}$ área suelo) del dosel del BST permite la optimización de la energía, lo que genera valores altos en las tasas de crecimiento e incrementa la competencia aumentando la mortalidad y el reclutamiento por la liberación de espacios de crecimiento. Por el contrario, en el BRM, que solo

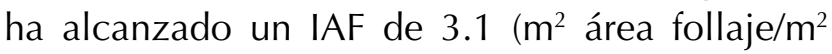
área suelo), el comportamiento funcional es inverso, los arboles maduros generan un dosel con baja superficie foliar, existe menos uso de la radiación y la tasa de crecimiento, mortalidad y reclutamiento presenta valores menores en comparación con las sucesiones tempranas. El IAF más alto en BST (8.3) y BSR (5.1) conduce a las más bajas intensidades en la RFA (16 a $18 \mu$ moles fotones. $\mathrm{m}^{2} . \mathrm{s}^{-1}$ ), como consecuencia del autosombreamiento, que obedece a la arquitectura planófila, la cual es definida como el arreglo de hojas y ramas insertas en ángulos que fluctúan entre $0^{\circ}$ y $30^{\circ}$ de esas dos comunidades, expresado por los $\mathrm{K}=0.75$ y 0.63 respectivamente. Para los bosques que se encuentran en un estado sucesional más avanzado (BRM y BSM), los cuales presentan IAF de 3.1 y 4.5 , adicional a los $K=0.49$ y 0.53 , arquitectura plagiófila (arreglos de hojas y ramas insertas en ángulos que

Tabla 2. Valores de diversidad intercomunitaria para cuatro tipos de bosque secundario en el Alto Magdalena.

\begin{tabular}{crrrrr}
\hline COMPARACIÓN & SR & JC & PD & PR & DE \\
\hline BST-BSR & 0.42 & 0.48 & 37.26 & 48.43 & 22.67 \\
BST-BRM & 0.18 & 0.25 & 59.37 & 75.33 & 36.77 \\
BST-BSM & 0.33 & 0.37 & 45.93 & 52.57 & 23.92 \\
BSR-BRM & 0.21 & 0.29 & 51.47 & 63.71 & 30.73 \\
BSR-BSM & 0.53 & 0.59 & 29.82 & 37.39 & 19.32 \\
BRM-BSM & 0.23 & 0.27 & 53.31 & 75.05 & 31.32 \\
\hline
\end{tabular}

BST: bosque secundario temprano. BSR: bosque secundario en recuperación. BRM: bosque ribereño maduro. BSM: bosque secundario maduro. SR: similaridad de Sorensen. JC: similaridad de Jaccard. PD: Porcentaje de disimilitud. PR: porcentaje de remotidad. DE: distancia euclidiana. 
fluctúan entre $30^{\circ}$ y $60^{\circ}$ ), el autosombreamiento es menor, lo que conduce a mayores intensidades de RFA de 22 a $25 \mu$ moles fotones. $\mathrm{m}^{2} . \mathrm{s}^{-1}$ (tabla 1 ).

La tabla 3 caracteriza el hábitat lumínico y lo vincula con la estructura, la diversidad y la dinámica del bosque a partir de una matriz de correlación, para los parámetros índice de área foliar (IAF), Coeficiente de extinción lumínica $(K)$, radiación fotosintéticamente activa (RFA), índice de diversidad de la serie logarítmica (Alfa), área basal en $\mathrm{m}^{2} \mathrm{ha}^{-1}(\mathrm{G})$, riqueza de especies de Margalef
(DMg), tasa media de crecimiento (TC), tasa anual de mortalidad $(M)$ y tasa anual de reclutamiento (R). Se observan altos valores de correlación entre el IAF, que expresa la magnitud de la cobertura del dosel y el papel en la captura y absorción de radiación, frente a la diversidad florística, las tasas de crecimiento medio, la mortalidad y el reclutamiento. Los valores negativos en la correlación con la diversidad indican que en bosques con doseles que tienen gran superficie foliar (IAF) para capturar la poca disponibilidad de RFA. La riqueza

Tabla 3. Matriz de correlación entre el hábitat lumínico, la estructura, la diversidad y la dinámica de los bosques secos tropicales.

\begin{tabular}{|c|c|c|c|c|c|c|c|c|c|}
\hline & IAF & K & RFA & Alfa & G & DMg & TC & $M \%$ & $\mathbf{R} \%$ \\
\hline \multirow{3}{*}{ IAF } & & 0.9714 & -0.9002 & -0.8918 & -0.4534 & -0.8085 & 0.9696 & 0.9838 & 0.9121 \\
\hline & & 100 & 100 & 100 & 100 & 100 & 100 & 100 & 100 \\
\hline & & 0.0286 & 0.0998 & 0.1082 & 0.5466 & 0.1915 & 0.0304 & 0.0162 & 0.0879 \\
\hline \multirow[t]{3}{*}{ K } & 0.9714 & & -0.9568 & -0.9702 & -0.4562 & -0.9041 & 0.9955 & 0.9885 & 0.9664 \\
\hline & 100 & & 100 & 100 & 100 & 100 & 100 & 100 & 100 \\
\hline & 0.0286 & & 0.0432 & 0.0298 & 0.5438 & 0.0959 & 0.0045 & 0.0115 & 0.0336 \\
\hline \multirow[t]{3}{*}{ RFA } & -0.9002 & -0.9568 & & 0.9242 & 0.6651 & 0.8197 & -0.9262 & -0.9034 & -0.9994 \\
\hline & 100 & 100 & & 100 & 100 & 100 & 100 & 100 & 100 \\
\hline & 0.0998 & 0.0432 & & 0.0758 & 0.3349 & 0.1803 & 0.0738 & 0.0966 & 0.0006 \\
\hline \multirow[t]{3}{*}{ Alfa } & -0.8918 & -0.9702 & 0.9242 & & 0.3307 & 0.9756 & -0.9744 & -0.951 & -0.9349 \\
\hline & 100 & 100 & 100 & & 100 & 100 & 100 & 100 & 100 \\
\hline & 0.1082 & 0.0298 & 0.0758 & & 0.6693 & 0.0244 & 0.0256 & 0.049 & 0.0651 \\
\hline \multirow[t]{3}{*}{ G } & -0.4534 & -0.4562 & 0.6651 & 0.3307 & & 0.1242 & -0.3706 & -0.3536 & -0.6413 \\
\hline & 100 & 100 & 100 & 100 & & 100 & 100 & 100 & 100 \\
\hline & 0.5466 & 0.5438 & 0.3349 & 0.6693 & & 0.8758 & 0.6294 & 0.6464 & 0.3587 \\
\hline \multirow[t]{3}{*}{ DMg } & -0.8085 & -0.9041 & 0.8197 & 0.9756 & 0.1242 & & -0.9261 & -0.8999 & -0.8353 \\
\hline & 100 & 100 & 100 & 100 & 100 & & 100 & 100 & 100 \\
\hline & 0.1915 & 0.0959 & 0.1803 & 0.0244 & 0.8758 & & 0.0739 & 0.1001 & 0.1647 \\
\hline \multirow[t]{3}{*}{$\mathrm{TC}$} & 0.9696 & 0.9955 & -0.9262 & -0.9744 & -0.3706 & -0.9261 & & 0.9959 & 0.939 \\
\hline & 100 & 100 & 100 & 100 & 100 & 100 & & 100 & 100 \\
\hline & 0.0304 & 0.0045 & 0.0738 & 0.0256 & 0.6294 & 0.0739 & & 0.0041 & 0.061 \\
\hline \multirow[t]{3}{*}{$\mathrm{M} \%$} & 0.9838 & 0.9885 & -0.9034 & -0.951 & -0.3536 & -0.8999 & 0.9959 & & 0.9177 \\
\hline & 100 & 100 & 100 & 100 & 100 & 100 & 100 & & 100 \\
\hline & 0.0162 & 0.0115 & 0.0966 & 0.049 & 0.6464 & 0.1001 & 0.0041 & & 0.0823 \\
\hline \multirow[t]{3}{*}{$\mathrm{R} \%$} & 0.9121 & 0.9664 & -0.9994 & -0.9349 & -0.6413 & -0.8353 & 0.939 & 0.9177 & \\
\hline & 100 & 100 & 100 & 100 & 100 & 100 & 100 & 100 & \\
\hline & 0.0879 & 0.0336 & 0.0006 & 0.0651 & 0.3587 & 0.1647 & 0.061 & 0.0823 & \\
\hline
\end{tabular}

IAF: índice de área foliar. K: Coeficiente de extinción lumínica. RFA: radiación fotosintéticamente activa. Alfa: índice de diversidad de la serie logarítmica. G: área basal en $\mathrm{m}^{2} \mathrm{ha}^{-1}$. DMg: riqueza de especies de Margalef. TC: tasa media de crecimiento. M\%: tasa anual de mortalidad. R\%: tasa anual de reclutamiento. 
de especies es baja. Sotobosques más iluminados permiten el establecimiento de más especies por unidad de área y hay más heterogeneidad de nichos de crecimiento. Igualmente, se detectó correlación negativa entre el IAF y la supervivencia de la comunidad arbórea. El área basal tiene baja correlación con el hábitat lumínico debido principalmente a la influencia de los grandes árboles del BRM que comparten el mismo hábitat de árboles de menores dimensiones de los bosques secundarios. La mortalidad correlaciona en forma positiva con el IAF y K, caso contrario con RFA. El reclutamiento tiene una tendencia similar.

\section{DISCUSIÓN}

Los bosques evaluados en general muestran una crono-secuencia que permite el incremento de los valores de la diversidad y la complejidad estructural que ofrece la sucesión de las coberturas naturales del bosque seco tropical del Alto Magdalena como lo expresado por Mendoza (1999), en estudios preliminares en fragmentos de bosque seco tropical de la costa caribe y valle del Magdalena. Los valores de los parámetros estructurales y de la riqueza de especies, heterogeneidad y rareza de especies (tabla 1) para BST, BSR y BSM son relativamente más altos que los encontrados por Fernández et al. (2014) para el sur del departamento del Tolima, zona que manifiesta un clima mas seco $(1350 \mathrm{~mm}$ de precipitación media anual). Lo que está acorde con lo demostrado por Gentry (1988; 1995), quien afirma que la riqueza de especies está asociada a la disponibilidad de humedad del medio. En cuanto al BRM, la riqueza de especies es la más alta reportada para este tipo de cobertura en áreas de bosque seco tropical ya que estudios realizados por Linares \& Fandiño (2009), Cabrera \& Galindo (2006) y Etter (1993) registran una menor densidad de especies en áreas de estudio similares tanto en el Valle del Cauca como en la costa atlántica.

En cuanto a la diversidad intercomunitaria (tabla 2), el complejo sucesional de los bosques secundarios manifiestan cambios graduales de la composición florística, asociados al desarrollo sucesional al interrumpir la actividad antrópica. Este incremento de especies en correlación con la recuperación de los servicios ecosistémicos y la funcionalidad del bosque ha sido estudiado por Kalascka et al. (2004) en bosques secos mesoamericanos.

Las diferencias encontradas en las tasas de crecimiento $(T C)$, mortalidad $(M)$ y reclutamiento $(R)$ para los cuatro tipos de bosque seco tropical que se muestran en la tabla 1 son altas en comparación con estudios similares realizados en bosques secos tropicales en Nicaragua (Flynn et al., 2011), lo cual puede estar asociado tanto a la orografía como a la disponibilidad de humedad, que para el flanco oriental de la cordillera central están asociados al efecto sombra de cordillera que incrementa el valor de la humedad relativa, generando un mejor entorno de crecimiento.

El crecimiento del árbol individual y del bosque en su conjunto depende en gran medida de su funcionalidad, es decir, de la forma como se obtienen los recursos que ofrece el entorno ambiental y de cómo los utilizan, siendo los principales la luz y el agua en el suelo (Burkhart \& Tomé, 2012), lo cual es expresado en la distribución y calidad del dosel. Lo que influye directamente en la acumulación de la biomasa, cuya distribución diferencial en sus componentes estructurales varía de acuerdo con las relaciones de competencia generadas por los árboles vecinos ubicados en su espacio vital (Trinder et al., 2013). En cuanto a los valores de las variables que describen el hábitat lumínico y su correlación con la dinámica del bosque (tabla 3), para las cuatro coberturas evaluadas (BST, BSR, $B S M, B R M)$ es claro el efecto directo que tienen el IAF, K y RFA sobre el crecimiento, la mortalidad y el reclutamiento. De tal manera que las mayores tasas de mortalidad y reclutamiento se generan bajo doseles con altos valores de IAF y máximos $\mathrm{K}$. Es decir, hay dependencia del autosombreado debido a la mayor superficie foliar del dosel y la arquitectura que este describe que inciden en la captura de la radiación y en la consecuente productividad 
de la comunidad forestal, esto afecta directamente tanto la estructura de las comunidades vegetales como su diversidad florística (Pretzsch, 2014), relaciones similares entre funcionalidad y tipos de cobertura han sido encontrados por Sterck et al. (2005), Craine \& Dybzinski (2013), lo cual sustenta los presentes resultados para los bosques secos tropicales del Alto Magdalena.

\section{CONCLUSIONES}

Existe relación directa entre la disponibilidad del recurso lumínico y la dinámica del bosque seco tropical. Las mayores tasas de crecimiento del BST están asociadas a hábitats con alta disponibilidad de RFA que es absorbida por un dosel con alto IAF, dando como consecuencia un alto crecimiento que genera un incremento en las tasas de mortalidad. Los espacios liberados son ocupados por nuevos individuos que esperan la oportunidad de crecimiento. Para sucesiones más avanzadas como los BSM el dosel que caracteriza la estructura del bosque tiene un menor IAF, lo que permite mayor disponibilidad de recursos en el sotobosque permitiendo una mayor diversidad de hábitats que son ocupados por varios tipos de especies, de ahí la mayor heterogeneidad y complejidad estructural. La consecuencia es un menor crecimiento y mayor estabilidad entre la mortalidad y el reclutamiento, los cuales deben su dinámica a factores endógenos a la comunidad biótica.

\section{AGRADECIMIENTOS}

Lo autores agradecen la valiosa colaboración de la Corporación Autónoma Regional del Tolima (Cortolima) y de la oficina de investigaciones de la Universidad del Tolima por el apoyo financiero otorgado para desarrollar la presente investigación, a través de la alianza estratégica Corporación-Universidad.

\section{REFERENCIAS BIBLIOGRÁFICAS}

Bazzaz, F., \& Pickett, S. (1980). Physiological ecology of tropical succession: a comparative review. Annual Review of Ecology, Evolution, and Systematics, 11, 287-310.

Burkhart, H., \& Tomé, M. (2012). Modeling forest trees and stand. Berlín: Springer-Verlag. 457 p.

Burnham, K. (1997). Distributional results for special cases of the Jolly-Seber model. Communications in Statistics, 26,1395-1409.

Cabrera, E., \& Galindo, G. (2006). Aproximación metodológica para la delimitación de ecosistemas de enclaves secos. Caso piloto: Cañones del río Dagua y del río Tuluá, Valle del Cauca - Colombia. Bogotá, D. C.: Instituto de Investigación de Recursos Biológicos Alexander von Humboldt. 105 p.

Carson, W., \& Schnitzer, S. (2008). Tropical forest community ecology. Wiley: Blacwell. 517 p.

Castro, G., Nigard, R., Gonzalez, B., \& Oden, P. (2005). Stand dynamics and basal area change in a tropical dry forest reserve in Nicaragua. Forest Ecology and Management, 208. 63-75.

Chazdon, R., \& Denslow, J. (2002). Floristic composition and species richness. En Chazdon, R., \& Whitmore, T. (Eds.), Foundations of Tropical Forest Biology (pp. 513-522). Chicago: Classic Papers with Commentaries, University of Chicago Press.

Colwell, R., Chao, A., Gotelli, N., Lin, S., Mao, C., Chazdon, R., \& Longino, J. (2012). Models and estimators linking individual-based and sample-based rarefaction, extrapolation, and comparison of assemblages. Journal of Plant Ecology, 5, 3-21

Craine, J., \& Dybzinski, R. (2013). Mechanisms of plant competition for nutrients, water and light. Functional Ecology, 27, 833-840.

Etter, A. (1993). Diversidad ecosistémica en Colombia hoy (pp. 43-61). En Nuestra diversidad biótica. Bogotá: CEREC y Fundación Alejandro Ángel Escobar.

Fernández, F., Melo, O., Álvarez, E., Perez, U., \& Lozano, A. (2014). Status of Knowledge, conservation and management of tropical dry forest in the Magdalena river Valley, Colombia. En Sanchez-Asofeifa, A., Power, J., Fernandes, G., \& Quesada M. 
(eds.). Tropical dry forest in the Americas (pp. 3568). CRC Press. Taylor \& Francis Group.

Flynn, B., Mirotchnick, N., Jain, M., Palmer, M., \& Naeem, S. (2011). Functional and phylogenetic diversity as predictors of biodiversity-ecosystem-function relationships. Ecology, 92(8), 1573-1581.

Gentry, A. (1988). Changes in plant community diversity and floristic composition on environmental and geographical gradients. Annals of the Missouri Botanical Garden, 75, 1-34.

Gentry, A. (1995). Diversity and floristic composition of neotropical dry forest. en: S. Bullock, S., Medina, E., \& Mooney, H. (eds.). Tropical deciduous forest ecosystems (pp. 116-194). Cambridge: Cambridge University Press.

Instituto Alexander von Humboldt (IAVH). (2002). El bosque seco tropical (bs-t) en Colombia, programa de inventario de la biodiversidad (pp. 1-24). Grupo de exploraciones y monitoreo ambiental gema. Bogotá: Instituto Alexander von Humboldt.

Janzen, D. (1986). Tropical dry forests: the most endangered major tropical ecosystem. En Wilson, E.O. (ed.). Biodiversity (pp. 130-137). Washington D. C.: National Academy Press.

Kalascka, M., Sanchez-Asofeifa, G., Calvo-Alvarado, J., Quesada, M., Rivard, D., \& Jansen, D. (2004). Species composition, similarity and diversity in three successional stages of a seasonally dry tropical forest. Forest Ecology and Management, 200, 227-247.

Linares, R., \& Fandiño, M. (2009). Estado del bosque seco tropical e importancia relativa de su flora leñosa, islas de la Vieja Providencia y Santa Catalina, Colombia, Caribe suroccidental. Revista de la Academia Colombiana de Ciencias, 33(126), 1-12.

Lobo, J., Quesada, M., Stoner, K., Fuchs, E., Herrerias-Diego, Y., Rojas-Sandoval, J., \& Saborio-Rodriguez, G. (2003). Factors affecting phenological patterns of bombacaceous trees in seasonal forests in Costa Rica and Mexico. American Journal of Botany, 90 (7), 1054-1063.

Luttge, U. (2008). Physiological Ecology of Tropical Plants. $2^{\text {da }}$ ed. Berlin: Springer-Verlag. 458 p.

Magurran, A. (1988). Ecological Diversity and its Measurement. New York: Princeton University. 179 p.
Magurran, A. (2004). Measuring Biological diversity. Oxford: Blackwell Publishing. 256 p.

Magurran, A., \& McHill, B. (2011). Biological diversity: Frontiers in measurement and assessment. New York: Oxford University Press. 345 p.

Melo, O., \& Vargas, R. (2003). Evaluación ecológica y silvicultural de ecosistemas boscosos. Ibagué: Universidad del Tolima, CRQ, Carder, Corpocaldas, Cortolima. 235 p.

Mendoza, C. (1999). Estructura y riqueza florística del bosque seco tropical en la región Caribe y el vaIle del río Magdalena, Colombia. Caldasia, 21(1), 70-94.

Montagnini, F., \& Jordan C. (2005). Tropical forest ecology: The basis for conservation and management. Berlin: Springer Verlag. 295 p.

Mooney, H., Bullock, S., \& Medina, E. (1996). Introducción to tropical dry forest. En Bullock, S., \& Mooney, H., Medina, E. (eds.). Seasonally dry tropical forests (pp. 1-6). Cambridge: Cambridge University Press.

Murphy, P., \& Lugo, A. (1996). Dry forests of Central America and the Caribbean. En Mooney, H., \& Bullock, S. (eds.). Seasonally Tropical Forests (pp. 9-34). Cambridge: University of Cambridge Press.

Pallardy, S. (2008). Physiology of woody plants ( $3^{\text {rd }}$ ed.). San Diego: Academic Press, Elsevier. 454 p.

Phillips, O., Vasquez, R., Monteagudo, A., Baker T., \& Nunez, P. (2005). Large lianas as hyperdynamic elements of the tropical forest canopy. Ecology, 86(5), 1250-1258.

Prance, W. (2006). Tropical savannas and seasonally dry forests: an introduction. Journal of Biogeography, 33, 385-386.

Pretzch, H. (2014). Canopy space filling and tree crown morphology in mixed-species stands compared with monocultures. Forest Ecology and Management, 327, 251-264.

Quesada, M., \& Stoner, K. (2004). Threats to the conservation of the tropical dry forest in Costa Rica. In: Frankie, G., Mata, A., \& Vinson, S. (ed.) Biodiversity conservation in Costa Rica: learning the lessons in a seasonal dry forest (pp. 266-280). Berkeley (CA, U. S. A.): University of California Press. 
Repizzo, A., \& Devia, C. (2008). Árboles y arbustos del valle seco del río Magdalena y la región Caribe colombiana: su ecología y usos. Bogotá D. C.: Facultad de Estudios Ambientales y Rurales, Pontificia Universidad Javeriana. $120 \mathrm{p}$.

Sánchez-Azofeifa, A., Kalacska, M., Quesada, M., Calvo-Alvarado, J., Nassa, J., \& Rodríguez, J. (2005). Need for integrated research for a sustainable future in tropical dry forests. Conservation Biology, 19(2), 1-2.

Sterck, F., Schieving, F., Lemmens, A., \& Pons, T. (2005). Performance of trees in forest canopies: explorations with a bottom-up functional-structural plant growth model. New Phytologist 166(3), 827-843.
Trinder, C., Brooker, R., \& Robinson, D. (2013). Plant ecology's guilty little secret: understanding the dynamics of plant competition. Functional Ecology, 27, 918-929.

Universidad del Tolima. (2002). Informe técnico proyecto "'Caracterización biofísica de la Eco-región Estratégica del desierto de la Tatacoa y su área de influencia". Ibagué (Tolima, Colombia): Universidad del Tolima, Universidad Surcolombiana, Universidad de Cundinamarca, Cortolima, CAM. 250 p.

Weiskittel, A., Hann, D., Kershaw, J., \& Vanclay, J. (2011). Forest growth and yield modeling. Wiley-Blacwell. $415 \mathrm{p}$. 